\title{
Density-potential relation via the single-particle kinetic energy density for one-dimensional two- level fermion systems with application to Be-like atomic ions in the $(1 s)^{2}(2 s)^{2}$ configuration
}

\author{
I. A. Howard ${ }^{1}$ \\ ${ }^{1}$ Department of Chemistry (ALGC), Free University of Brussels (VUB), B-1050 Brussels, Belgium \\ N. H. $\operatorname{March}^{2,3,4}$ \\ ${ }^{2}$ Donostia International Physics Center (DIPC), E-20080 San Sebastian, Spain \\ ${ }^{3}$ Department of Physics, University of Antwerp, B-2020 Antwerp, Belgium \\ ${ }^{4}$ Oxford University, Oxford, England \\ (Received 30 May 2006; published 27 November 2006)
}

\begin{abstract}
Using the Dawson-March transformation of two-level wave functions in terms of density amplitude and phase, a differential equation is constructed relating the single-particle kinetic energy to the fermion density. Employing then the differential form of the virial theorem derived by March and Young [Nucl. Phys. 12, 237 (1959)], a nonlinear electron-density-one-body potential relation emerges. Application to the Be-like atomic ions is briefly considered.
\end{abstract}

DOI: $10.1103 /$ PhysRevA.74.054503

PACS number(s): 31.15.-p, 71.15.Mb

In early work, Dawson and March [1] set up a theory of two-level systems in terms of density amplitude and phase. Here, we employ their approach, but now restricted to onedimensional two-level Fermi systems, with the ground state characterized by single-particle wave functions $\psi_{1}(x)$ and $\psi_{2}(x)$, according to [1]

$$
\psi_{1}(x)=\rho^{1 / 2}(x) \cos [\theta(x)]
$$

and

$$
\psi_{2}(x)=\rho^{1 / 2}(x) \sin [\theta(x)]
$$

For the singly filled levels considered here until the final application to Be with configuration $(1 s)^{2}(2 s)^{2}$, it follows immediately from Eqs. (1) and (2) that the electron density $\rho(x)=\psi_{1}^{2}(x)+\psi_{2}^{2}(x)$.

If we define the kinetic energy density $t_{g}(x)$ corresponding to the positive-definite gradient $(g)$ wave-function form proportional to $(\nabla \psi)^{2}$, then March and Young [2] employed the equation of motion of the idempotent Dirac density matrix $\gamma\left(x, x^{\prime}\right)$, which is such that $\left.\gamma\left(x, x^{\prime}\right)\right|_{x^{\prime}=x}=\rho(x)$, to derive the so-called differential virial theorem in the form

$$
\frac{\partial t_{g}(x)}{\partial x}=-\frac{1}{2} \rho \frac{\partial V}{\partial x}+\frac{\rho(x)^{\prime \prime \prime}}{8}
$$

by expanding $\gamma\left(x, x^{\prime}\right)$ about its diagonal in powers of $(x$ $-x^{\prime}$ ). Multiplying Eq. (3) by $x$ and integrating over $x$ from $-\infty$ to $\infty$ readily yields the quantum-mechanical form of the virial theorem going back to Clausius. In Eq. (3), $V(x)$ is the one-body potential energy generating wave functions $\psi_{1}$ and $\psi_{2}$ in Eqs. (1) and (2) via the Schrödinger equation. Utilizing $\gamma\left(x, x^{\prime}\right)$ in terms of $\rho(x)$ and the phase $\theta(x)$, related via a nonlinear pendulum equation given in Eq. (7) below, the kinetic energy density $t_{g}(x)$ focused on below is given by [1]

$$
t_{g}(x)=\left.\frac{1}{2} \frac{\partial^{2} \gamma\left(x, x^{\prime}\right)}{\partial x \partial x^{\prime}}\right|_{x^{\prime}=x}=t_{W}(x)+\frac{1}{2} \rho \theta^{\prime 2}(x),
$$

where $t_{W}(x)$ is the von Weizsäcker inhomogeneity kinetic energy density defined by

$$
t_{W}(x)=\frac{\rho^{\prime 2}(x)}{8 \rho(x)} .
$$

In terms of the quotient $\rho^{\prime}(x) / \rho(x) \equiv Q(x)$, the pendulum equation already referred to reads [1]

$$
\theta^{\prime}(x)+Q(x) \theta^{\prime}(x)+\lambda \sin 2 \theta(x)=0 .
$$

What Eq. (6) tells us is that it will be simplest to regard the equation as the basis for deriving the quotient $Q(x)$ as a functional of the phase $\theta(x)$, which in turn is related to the kinetic energy density $t_{g}(x)$ via Eq. (4).

Below, we shall carry out such a program quite generally for the two-level case, but we have found it helpful in guiding such a calculation to refer immediately to a solvable model, namely the harmonic oscillator $(\mathrm{HO})$ in one dimension, which goes back to the study of Lawes and March [3]. These workers in fact derive a density-potential $(\rho-V)$ relation in the form of a third-order linear homogeneous differential equation for $\rho(x)$ in terms of $V(x)=x^{2} / 2$ and its first derivative. Their result is

$$
\rho^{\prime \prime \prime}(x) / 8+[N-V(x)] \rho^{\prime}(x)+\frac{1}{2} \frac{\partial V}{\partial x} \rho(x)=0,
$$

which is valid in fact for an arbitrary number $N$ of fermions filling the lowest levels singly, to be definite. In particular, this Eq. (7) must apply to the two-level case corresponding to $N=2$. But combining the $\mathrm{HO}$ result (7) with the general result (3), we can remove the third derivative of $\rho(x)$ from Eq. (7) to find 


$$
-\frac{\partial t(x)}{\partial x}+[N-V(x)] \rho^{\prime}(x)=0,
$$

where $t(x)$ is the counterpart of $t_{g}(x)$ in Eqs. (3) and (4), but now corresponding to the wave-function form proportional to $\psi \nabla^{2} \psi$. This exceedingly simple result relating $t^{\prime}(x) / \rho^{\prime}(x)$ to $[N-V(x)]$, we reiterate, is restricted to the HO potential energy $x^{2} / 2$, as is readily verified by using other specific forms of $V(x)$. But what we wish to emphasize in connection with the two-level case for a general potential energy $V(x)$ is that for the above example one can use Eq. (7) to find

$$
\frac{\rho^{\prime \prime \prime}(x)}{8}+t^{\prime}(x)+\frac{1}{2} \rho(x) \frac{\partial}{\partial x} \frac{t^{\prime}(x)}{\rho^{\prime}(x)}=0 .
$$

This is the desired relation between $\rho(x)$ and kinetic energy $t(x)$, but restricted to the $\mathrm{HO}$ example. In terms of $Q(x)$ $=\rho^{\prime} / \rho$, Eq. (9) is readily rewritten by removing $\rho^{\prime \prime \prime}(x)$ in favor of $Q(x)$ and its low-order derivatives, as

$$
\begin{gathered}
\frac{1}{2} \rho(x) \frac{\partial}{\partial x}\left(\frac{t^{\prime}(x)}{\rho^{\prime}(x)}\right)+\frac{t^{\prime}(x)}{\rho^{\prime}(x)}+\frac{Q^{\prime \prime}(x)}{\rho^{\prime}(x)}+\frac{2 \rho(x)}{\rho^{\prime}(x)} Q^{\prime}(x) \\
+\frac{\rho^{\prime \prime}(x)}{\rho^{\prime}(x)} Q(x)=0 .
\end{gathered}
$$

This is a linear first-order differential equation for the quotient $t^{\prime}(x) / \rho^{\prime}(x)$ in terms of the fermion density $\rho(x)$ and its low-order derivatives. Though Eq. (10) is restricted to the HO example, it motivates the ensuing derivation of such a kinetic energy density-fermion density relation for a twolevel system [e.g., Be in configuration $(1 s)^{2}(2 s)^{2}$, referred to briefly again below] generated by an arbitrary one-body potential $V(x)$.

To achieve this relation, we return to the nonlinear pendulum Eq. (6), where $\lambda$ is an eigenvalue related to the energy levels corresponding to eigenfunctions $\psi_{1}(x)$ and $\psi_{2}(x)$ in Eqs. (1) and (2). In principle, Eq. (6) allows us to write the fundamental relation $\theta=\theta[Q(x) ; \lambda]$. However, in the absence of knowledge of this functional, let us remove $\lambda$ from Eq. (6) by differentiation with respect to $x$. Then we obtain readily the further result that

$$
\frac{\theta^{\prime \prime}(x)}{\theta^{\prime}(x)}+Q^{\prime}(x)+Q(x) \frac{\theta^{\prime \prime}(x)}{\theta^{\prime}(x)}+2 \lambda \cos 2 \theta=0 .
$$

Taking another derivative of Eq. (11) then yields

$$
\frac{d}{d x} \frac{\theta^{\prime \prime}}{\theta^{\prime}}+Q^{\prime \prime}(x)+Q(x) \frac{d}{d x} \frac{\theta^{\prime}}{\theta^{\prime}}+Q^{\prime}(x) \frac{\theta^{\prime \prime}}{\theta^{\prime}}-4 \lambda \theta^{\prime} \sin 2 \theta=0 .
$$

Evidently Eq. (6) can now be used to remove $\lambda \sin 2 \theta$ from the final term on the left-hand side (LHS) of Eq. (12). Before writing the explicit result thereby obtained, let us now return to the kinetic energy density in Eqs. (4) and (5) and define the positive-definite quotient $q(x)$ by

$$
q(x)=\frac{t_{g}(x)-t_{W}(x)}{\rho(x)}=\frac{1}{2} \theta^{\prime 2}(x) .
$$

Differentiating this Eq. (13) yields

$$
\theta^{\prime} \theta^{\prime \prime}=q^{\prime}(x)
$$

and hence

$$
\frac{\theta^{\prime \prime}}{\theta^{\prime}}=\frac{q^{\prime}}{2 q},
$$

utilizing again Eq. (13). Substituting Eq. (15) into Eq. (12) gives almost immediately

$$
\begin{aligned}
& \frac{d}{d x} \frac{\theta^{\prime \prime}}{\theta^{\prime}}+Q^{\prime \prime}(x)+\frac{Q^{\prime}(x)}{2}\left(\frac{q^{\prime}}{q}\right)+\frac{Q(x)}{2} \frac{d}{d x}\left(\frac{q^{\prime}}{q}\right) \\
&+4\left[\theta^{\prime} \theta^{\prime \prime}+Q(x) \theta^{\prime 2}\right]=0 .
\end{aligned}
$$

Using again Eqs. (14) and (13), the last term on the LHS of Eq. (16) becomes $4\left[q^{\prime}+2 Q q\right]$. Therefore, Eq. (16) now depends on the phase $\theta(x)$ only through the first term. To remove this, let us differentiate Eq. (14) to obtain

$$
\theta^{\prime 2}+\theta^{\prime} \theta^{\prime \prime}=q^{\prime \prime} \text {. }
$$

Dividing both sides of Eq. (17) by $\theta^{2}$ and using Eqs. (13) and (15), it follows that

$$
\frac{\theta^{\prime \prime}}{\theta^{\prime}}=\frac{q^{\prime \prime}}{2 q}+\frac{q^{\prime 2}}{4 q^{2}} .
$$

Hence, returning to Eq. (16) - plus the content of the sentence following this equation-we have the following linear differential equation for the quotient $Q(x)$ :

$$
\begin{aligned}
Q^{\prime \prime}(x) & +\frac{Q^{\prime}(x)}{2} \frac{q^{\prime}}{q}+\left[\frac{1}{2} \frac{d}{d x}\left(\frac{q^{\prime}}{q}+8 q\right)\right] Q(x)+\left[\frac{1}{2} \frac{d^{2}}{d x^{2}}\left(\frac{q^{\prime}}{q}\right)\right. \\
& \left.+\frac{1}{4} \frac{d}{d x}\left(\frac{q^{\prime 2}}{q^{2}}\right)+4 q^{\prime}\right]=0 .
\end{aligned}
$$

Since, from Eq. (19), $q$ is determined from Eq. (6) by kinetic energy densities $t_{g}(x)$ and $t_{W}(x)$ plus the fermion density $\rho(x)$, it follows that Eq. (19) is the desired two-level relation between $\rho(x)$ and kinetic energy density. To relate the HO example to the general Eq. (19) requires use of the specific form of $\theta(x)$ for the two-level oscillator. Then one finds the two-level HO result that $q^{\prime} / q=-2 Q(x)-4 x$, which makes the quantity $(1 / 2) d^{2}\left(q^{\prime} / q\right) / d x^{2}+Q^{\prime \prime}(x)$ appearing on the lefthand side of Eq. (19) identically zero. The remainder of Eq. (19) is then an identity if one uses explicit HO forms, thereby confirming that Eq. (19) embraces Eq. (10). In fact, the integral form of the differential virial Eq. (3), namely

$$
t_{g}(x)=\frac{1}{2} \int_{x}^{\infty} \rho(s) \frac{\partial V(s)}{\partial s} d s-\frac{\rho^{\prime \prime}(x)}{8},
$$

can be used, in conjunction with Eq. (13), to convert Eq. (19) into a density-potential relation: a long-term aim, of course, of density-functional theory. However, as the detail proliferates, it is best to regard Eqs. (16), (13), (5), and (19) as determining simultaneously the exact density-potential relation for the general two-level potential energy $V(x)$.

A reviewer has asked us to stress two further points here. First, Eq. (19) may also be treated as the third-order nonlinear differential equation for the quantity $q(x)$. Then one can find $q(x)$ for a given $\rho(x)$, which defines $Q(x)$. After that, 
with both $\rho(x)$ and $q(x)$ known, one can calculate the kinetic energy density $t_{g}(x)$. The second point concerns the boundary conditions to be imposed on any numerical calculations using the present method. The Kato nuclear cusp condition will be applicable here; of course, one must have integrability through the entire system of the physical densities for finite systems, both the electron and kinetic energy densities.

As an application of the above, let us briefly consider the ground-state configuration $(1 s)^{2}(2 s)^{2}$ of the Be-like sequence of atomic ions with atomic number $Z$. We denote the wave functions generated by the one-body central potential $V(r)$ having the (as yet unknown) form

$$
V(r)=V_{\text {Hartree }}(r)+V_{\mathrm{xc}}(r),
$$

where $V_{\mathrm{xc}}(r)$ is the exchange-correlation potential of densityfunctional theory [4], by $\phi_{1 s}(r)$ and $\phi_{2 s}(r)$ to distinguish them from the one-dimensional wave functions $\psi_{1}(x)$ and $\psi_{2}(x)$ in Eqs. (1) and (2). The wave function $\phi_{1 s}(r)$ satisfies

$$
\frac{1}{r} \frac{d^{2}}{d r^{2}}\left(r \phi_{1 s}\right)+2\left[\epsilon_{1 s}-V(r)\right] \phi_{1 s}=0,
$$

with an analogous equation for $\phi_{2 s}$. Equation (22) is, of course, a one-dimensional Schrödinger equation for $\psi_{1}$ $\equiv r \phi_{1 s}$, so that the above one-dimensional analysis is immediately applicable to this Be sequence, given a form of $V(r)$. Alternatively, as in the recent study of Amovilli and March [5] on the Be atom itself, if the ground-state density $\rho(r)$ is calculated by the diffusion quantum Monte Carlo procedure, the analog of Eq. (15) plus Eq. (16) can be utilized to extract $V(r)$. This avoids appeal to off-diagonal behavior of the Dirac density matrix $\gamma\left(r, r^{\prime}\right)$, as is required in the procedure set out in Ref. [5].

In summary, Eq. (19) is the central result of the present study. It constitutes a second-order differential equation relating the kinetic energy density $t_{g}(x)$, via Eqs. (5) and (6), to the fermion density $\rho(x)$. This differential equation is characterized entirely by the fermion ground-state density $\rho(x)$ and its low-order derivatives. It is valid for a general form $V(x)$ of the common potential energy governing the fermion motion in the two-level system considered here.

N.H.M. wishes to acknowledge that his contribution to the present study was carried out during a visit to DIPC, San Sebastian. It is a pleasure to thank P.M. Echenique and A. Rubio for generous hospitality.
[1] K. A. Dawson and N. H. March, J. Chem. Phys. 81, 5850 (1984).

[2] N. H. March and W. H. Young, Nucl. Phys. 12, 237 (1959).

[3] G. P. Lawes and N. H. March, J. Chem. Phys. 71, 1007 (1979).
[4] R. G. Parr and W. Yang, Density Functional Theory of Atoms and Molecules (Oxford University Press, New York, 1989).

[5] C. Amovilli and N. H. March, Chem. Phys. Lett. 423, 94 (2006). 